\title{
Thermal Characteristics and Measurement of Nanoscale Materials
}

\author{
Taikyeong T. Jeong ${ }^{1}$ and Young Seok Song ${ }^{2, \star}$ \\ ${ }^{1}$ Department of Electrical and Computer Engineering \\ University of Delaware, Newark, DE 19716 USA \\ ttjeong@alumni.utexas.net \\ ${ }^{2}$ Center for Composite Materials \\ University of Delaware, Newark, DE 19716 USA \\ youngseoks@gmail.com
}

\begin{abstract}
Numerical prediction of the physical properties of nanocomposites is an attractive area that requires more discussion and investigation. In this paper, we calculate the thermal conductivity of nanocomposites embedded with carbon nanotubes (CNTs) based on the representative volume element (RVE) concept. The RVE, which encompasses a single CNT, was constructed assuming that the CNTs are distributed in polymeric material homogeneously, and also assuming that the CNTs have no interaction with other CNTs. This research describes the thermal characteristics of nanoscale materials - CNTs filled nanocomposites - as a case study and measured their thermal conductivity, for the purpose of validation of numerical results. The dispersion state of the CNTs was observed using field emission scanning electronic microscope (FESEM). We found that the numerically predicted thermal conductivity is closely matches the experimental one and that the numerical tool employed in the study is superior to other analytical and numerical methods.
\end{abstract}

\section{Introduction}

Polymeric nanocomposites filled with nanoscale particles such as clay, nanofiber, and carbon nanotubes (CNTs) have been vigorously investigated over the past decade and remarkable achievements have been reported [1. It was recently reported that CNT nanocomposites possess excellent thermal conductivities which can be applied to production of miniature devices managing heat transfer. In this paper, we focus on the thermal characteristics of the CNT filled polymeric composites but not thermal conductivity of the CNT itself. Most studies on thermal conductivity of CNT nanocomposites have been carried out experimentally or analytically 2. To consider the contribution of various determinants like CNT geometry or concentration on the thermal conductivity, numerical analysis and calculation is required. In the current study, the effective thermal conductivity tensor of CNT filled composites is discussed using a homogenization method. In

\footnotetext{
^ Manuscript Dec. 12, 2005; T. Jeong was with the University of Texas at Austin and the corresponding author is Y. Song.
} 
particular, the influence of the aspect ratio and the content of CNTs are investigated. In contrast to most of the previous studies on the homogenization method using the FEM, control volume finite element method (CVFEM) is employed in the implementation of the homogenization method. The bounding approach is also adopted to take the orientation state of the CNTs into account and the analytic model [3] is used in the comparison with numerical results. The predicted thermal conductivities of nanoscale materials are compared with experimental results.

\section{Computation Method}

The AEH method is superior in several ways to other analytic and numerical methods. The contribution of complex geometries and anisotropic material properties of fillers can be precisely calculated through the AEH method as well as both localization and homogenization for heterogeneous materials. In the current study, two different packing structure of the CNTs, square and hexagonal packing, are taken into account so as to evaluate the contribution of CNT packing configuration as shown in Fig. 1 (a) and (b), respectively.

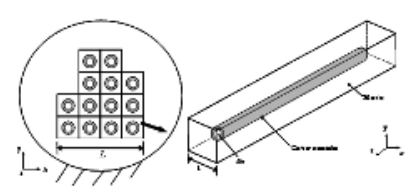

(a)

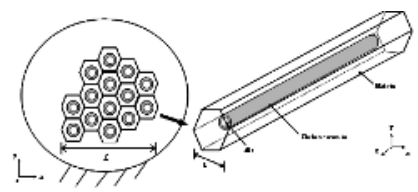

(b)

Fig. 1. Schematic illustration of (a) square packed RVE and (b) hexagonally packed RVE in CNT filled nanocomposites

The periodic unit cell consists of three different regions, i.e., matrix, CNT, and air which are denoted by $\Omega_{m}, \Omega_{c}$, and $\Omega_{a}$, respectively. Each region has its own thermal conductivity. This study assume the following: (i) CNTs are homogeneously dispersed in the CNT/polymer composites and have uniform dimensions including their length, inner, and outer diameters. (ii) there is no direct interaction between the adjacent CNTs. (iii) the CNT composites contain the periodic unit cell which includes a single CNT embedded unidirectionally. The scale parameter, $\varepsilon=\frac{l}{L} \ll 1$, is defined as the ratio of the characteristic length scales, which implies that the entire dimension, $\Omega$, of RVE is negligible compared to the characteristic length, $L$, for all of the composites. The temperature field is asymptotically expanded as below.

$$
T\left(x_{i}, y_{i}\right)=T^{(0)}\left(x_{i}, y_{i}\right)+\varepsilon T^{(1)}\left(x_{i}, y_{i}\right)+\varepsilon^{2} T^{(2)}\left(x_{i}, y_{i}\right)+\ldots
$$

where $x_{i}$ and $y_{i}$ indicate the global and local length scales. The asymptotic expansion homogenization method $(\mathrm{AEH})$ adopts two distinct coordinate systems, i.e., macroscopic scale $x_{i}$ and microscopic scale $y_{i}$, which can describe the 
macroscopic and microscopic behaviors of CNT filled composites. It is assumed that the temperature field is periodic with respect to $y_{i}$ coordinate system. The global coordinate system and the local coordinate system have the relation of $y_{i}=\frac{x_{i}}{\varepsilon}$. The differential operator is written as the following.

$$
\frac{\partial}{\partial y_{i}}=\frac{\partial}{\partial y_{i}}+\varepsilon \frac{\partial}{\partial x_{i}}
$$

In the steady-state heat conduction problem, the governing equation for each region is shown as below.

$$
\begin{array}{r}
-\frac{\partial}{\partial x_{i}}\left[k_{i j}{ }^{m} \frac{\partial T}{\partial x_{j}}\right]=f_{m} \quad \text { in } \Omega_{m} \\
-\frac{\partial}{\partial x_{i}}\left[k_{i j}{ }^{c} \frac{\partial T}{\partial x_{j}}\right]=f_{c} \quad \text { in } \Omega_{c} \\
-\frac{\partial}{\partial x_{i}}\left[k_{i j}{ }^{a} \frac{\partial T}{\partial x_{j}}\right]=f_{a} \quad \text { in } \Omega_{a}
\end{array}
$$

where $k_{i j}{ }^{m}, k_{i j}{ }^{c}$, and ${k_{i j}}^{a}$ are the second order thermal conductivity tensors for matrix, CNT, and air, respectively. $f_{m}, f_{c}$, and $f_{a}$ denote the volumetric heat generation for each region. In this study, the heat generation is not considered and the thermal conductivity tensor is assumed to be independent of temperature. After substituting the equation (1) into the equations (3)-(5), the following equations are obtained by collecting the terms with the same order of $\varepsilon$.

For $1^{\text {th }}$ order of $\varepsilon$,

$$
\begin{array}{rr}
-\frac{\partial}{\partial y_{i}}\left[k_{i j}{ }^{m} \frac{\partial T^{1}}{\partial y_{j}}\right]-\frac{\partial}{\partial y_{i}}\left[k_{i j}{ }^{m} \frac{\partial T^{0}}{\partial x_{j}}\right]-\frac{\partial}{\partial x_{i}}\left[k_{i j}{ }^{m} \frac{\partial T^{0}}{\partial y_{j}}\right] & \text { in } \Omega_{m} \\
-\frac{\partial}{\partial y_{i}}\left[k_{i j}{ }^{c} \frac{\partial T^{1}}{\partial y_{i}}\right]-\frac{\partial}{\partial y_{i}}\left[k_{i j}{ }^{c} \frac{\partial T^{0}}{\partial x_{j}}\right]-\frac{\partial}{\partial x_{i}}\left[k_{i j}{ }^{c} \frac{\partial T^{0}}{\partial y_{j}}\right] & i n \Omega_{c} \\
-\frac{\partial}{\partial y_{i}}\left[k_{i j}{ }^{a} \frac{\partial T^{1}}{\partial y_{i}}\right]-\frac{\partial}{\partial y_{i}}\left[k_{i j}{ }^{a} \frac{\partial T^{0}}{\partial x_{j}}\right]-\frac{\partial}{\partial x_{i}}\left[k_{i j}{ }^{a} \frac{\partial T^{0}}{\partial y_{j}}\right] & i n \Omega_{a}
\end{array}
$$

The perturbation temperature, $T^{1}$, is assumed to be

$$
T^{1}\left(x_{i}, y_{i}\right)=-\chi_{j}\left(y_{i}\right) \frac{\partial T^{0}}{\partial x_{j}}\left(x_{i}\right)+\tilde{T}^{1}\left(x_{i}\right)
$$

where $\chi_{j}$ is the arbitrary characteristic function which is independent of $x_{i}$ coordinate system and has periodicity with respect to $y_{i}$ coordinate system. As a result, the final governing equations are obtained by substituting the equation (12) into the equations (9)-(11) as below.

$$
\begin{aligned}
& -\frac{\partial}{\partial x_{i}}\left[k_{i k}{ }^{m} \frac{\partial \chi_{j}}{\partial y_{k}}\right]=\frac{\partial}{\partial y_{i}} k_{i j}{ }^{m} \quad \text { in } \Omega_{m} \\
& -\frac{\partial}{\partial x_{i}}\left[k_{i k}{ }^{c} \frac{\partial \chi_{j}}{\partial y_{k}}\right]=\frac{\partial}{\partial y_{i}} k_{i j}{ }^{c} \quad \text { in } \Omega_{c} \\
& -\frac{\partial}{\partial x_{i}}\left[k_{i k}{ }^{a} \frac{\partial \chi_{j}}{\partial y_{k}}\right]=\frac{\partial}{\partial y_{i}} k_{i j}{ }^{a} \quad \text { in } \Omega_{a}
\end{aligned}
$$


The homogenized effective conductivity tensor is calculated by integrating the conductivity tensor over the whole domain, $\Omega$, as given by the following equation.

$$
<k_{i j}>=\frac{1}{\Omega} \int_{\Omega}\left[k_{i j}-k_{i k} \frac{\partial \chi_{j}}{\partial y_{k}}\right] d y
$$

\section{Numerical Implementation}

The control volume finite element method (CVFEM), which is known as a very efficient numerical method for heat transfer problems is employed as numerical implementation method in this study. Equations (13)-(15) are rewritten in the general form as below.

$$
\frac{\partial J_{i}}{\partial y_{i}}=S
$$

where $J_{i}$ is the diffusion flux and $S$ is the source term. In the matrix region, $\Omega_{m}$, the diffusion flux and the source term are given by

$$
\begin{gathered}
J_{i}=-k_{i k} m \frac{\partial \chi_{j}}{\partial y_{k}}+k_{i k}{ }^{m} \\
S=0
\end{gathered}
$$

The diffusion flux and the source term for the other regions are obtained in ways similar to the above equations. Because CVFEM is based on the conservation principle within the control volume, we integrate equation (17) over the control volume as the following equation.

$$
\int_{\partial V} J_{i} n_{i} d s=\int_{\partial V} S d V
$$

where $\partial V$ is the surface of control volume, and $n_{i}$ is the outward unit vector normal to a differential area, $d s$. In order to integrate equation (20), specification of the interpolation function for the dependent variable, $\chi$, is needed in each element. The linear interpolation function is employed for the characteristic function in the current study. Substitution of the interpolation function into equation (20) yields the complete set of discretization equations. More details on the numerical analysis procedure were presented in the previous study.

The unit cells with unidirectionally aligned CNTs have transversely isotropic physical properties, but the CNTs are dispersed randomly in the composites. Therefore, the effective thermal conductivity for a composite filled with randomly dispersed CNTs is obtained from an orientation averaging procedure. In order to describe the orientation state of CNTs, the second order orientation tensoris adopted. In addition, to validate the numerically predicted thermal conductivities, they are compared with the results obtained by the analytic model proposed by Lewis and Nielsen [3]. This model can explain the effect of the shape and the packing type of the particles for two phase systems. However, it does not take into account the anisotropy of thermal conductivity for CNTs and the contribution of air within the CNT. 


\section{Measurement}

In order to obtain good and homogeneous dispersion of the CNTs in the epoxy resin, acid treatment on the CNTs was carried out, which also removed impurities including amorphous carbons, graphite particles, and metal catalysts. The CNTs were treated in a 3-to- 1 mixture of $65 \%$ H2SO4/HNO3, under the condition of $100 \mathrm{C}$ for $30 \mathrm{~min}$., after which they were washed with distilled water and then dried in a vacuum oven. The treated CNTs were then dispersed in a solvent and mixed with the epoxy resin. After evaporating the solvent, the hardener was added to the mixture with the CNT loading of 0.5 and $1.0 \mathrm{wt} . \%$. The epoxy and CNT mixture was cast into a cylindrical cavity and cured in a vacuum oven. The specimen had a diameter of $10 \mathrm{~mm}$ and length of $20 \mathrm{~mm}$. Therefore, we measured the thermal conductivity measurement by employing ASTM E1225-87, which was based on temperature difference between the reference and the specimen at steady state and room temperature. For verification of uniform dispersion of the CNTs, morphological characterization was performed by using an FESEM.

\section{Results and Discussion}

Fig. 2 presents the increase of thermal conductivity of nanocomposites filled with 0.5 wt. \% CNTs with respect to the CNT aspect ratio. When the CNTs are hexagonally arranged in the nanocomposites, the nanocomposites have higher thermal conductivities. As the aspect ratio of CNTs increases, thermal conductivities of the nanocomposites with hexagonal or square packing of the CNTs, eventually approaching a limiting value, which can be determined by the rule for mixture of conductivities for distinct regions. From here, the thermal conductivities predicted numerically mean the values calculated for the square packing of the CNT.

In Fig. 3, the thermal conductivities calculated by the analytic model and the homogenization technique are compared in the axial and the parallel directions of the CNT when the CNT loading is 0.5 wt. \% (0.29\% volume fraction). In order to vary the aspect ratio of CNTs, length of the CNT in the RVE is altered. In the case of the axial direction of the $\mathrm{CNT}$, both analytic and numerical predictions

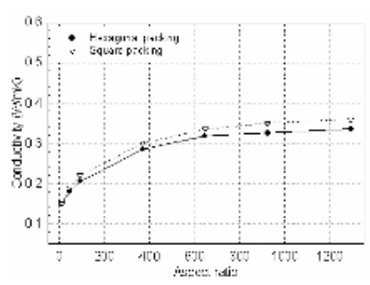

Fig. 2. Effect of packing the configuration of the CNTs' thermal conductivities in the axial direction when the CNTs of 0.5 wt. $\%$ are filled in nanocomposites 

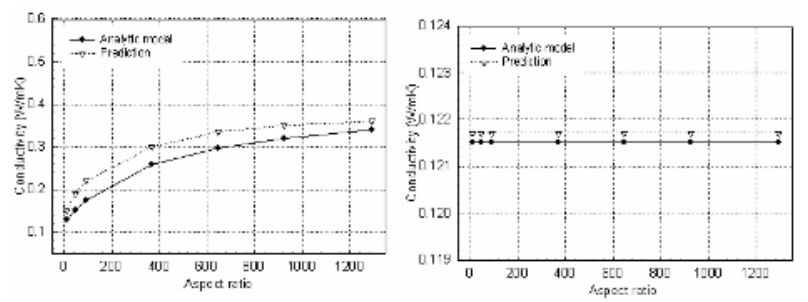

Fig. 3. Effective thermal conductivities with respect to aspect ratio of CNT (a) in the axial direction and (b) in the parallel directions

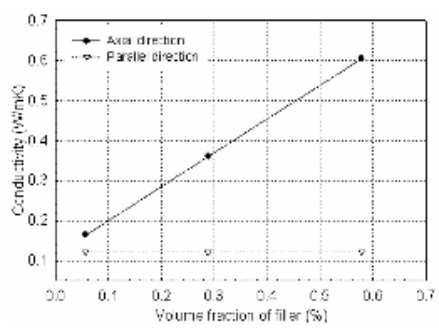

Fig. 4. Effective thermal conductivities calculated numerically with respect to CNT volume fraction

are increased with on increase in the aspect ratio. The thermal conductivity obtained by the homogenization method increases more rapidly with respect to the aspect ratio than that obtained the analytic model. When the CNT length is relatively large, the homogenization method is closer to material properties averaged by the rule of mixture with the volume fraction. On the other hand, it is shown in Fig. 3 (b) that thermal conductivities in the parallel direction of the CNTs have little variation with respect to the aspect ratio of CNT. The effect of the CNT volume fraction on the thermal conductivity is examined as shown in Fig. 4 and Fig. 5. Therefore, Fig. 4 shows that the thermal conductivities of the nanocomposites in the axial direction are much higher compared to the parallel direction and that the difference between them becomes large as the volume fraction of the CNT increase.

In addition, Fig. 5 (a) presents the effective thermal conductivity of composites filled with randomly oriented CNTs. The experimental measurement was carried out for CNT volume fractions of 0.29 and $0.58 \%$. The numerical and experimental data for higher volume fraction of CNTs are slightly different. The discrepancy has two causes. First, in the case that the volume fraction is relatively high, it is difficult to reach the homogeneous dispersion without aggregation of the CNTs, as was assumed for this study. Second, the bounding approach using the orientation tensor cannot completely take into account the contribution of the randomly oriented CNTs. Fig. 5 (b) shows an FESEM image of the composite filled with CNTs of 0.5 wt. \%. The image indicates that the embedded CNTs are homogeneously dispersed in the epoxy resin and direct contact between 

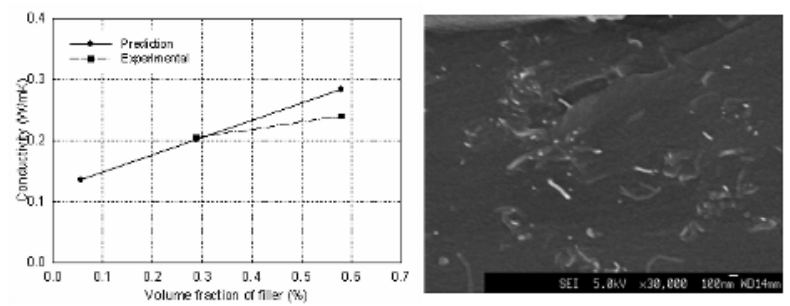

Fig. 5. (a) Effective thermal conductivity for the composites filled with randomly dispersed CNTs with respect to CNT volume fraction and (b) morphological observation of CNTs by FESEM

neighboring CNTs is negligible. This figure confirms the assumption that there is no direct interaction between the adjacent CNTs. Although the CNT loading is too small to reach the electrical percolation, mechanical and rheological properties of CNT filled composites can be enhanced dramatically [1. On the other hand, it has been shown that thermal conductivities do not show the percolation behavior in contrast to electrical conductivities [6].

\section{Conclusion}

The current study shows that the homogenization technique is a promising tool because it can consider such anisotropic material properties as thermal conductivity, elastic modulus, and thermal expansion coefficient as well as geometrical complexity. Specifically speaking, the effective thermal conductivity of the composites filled with CNTs is evaluated by the homogenization technique. The control volume finite element method (CVFEM) is employed so as to implement the homogenization method. The two different RVE structures filled with a single CNT are adopted in calculation of the thermal conductivity of composites. It is assumed that the composites have geometric periodicity with respect to local length scale. Orientation of the CNTs embedded in the composites is taken into account using the bounding approach. The effect of the aspect ratio and volume fraction of CNTs on the effective thermal conductivity was investigated. The numerically calculated conductivities closely match the experimental result.

\section{References}

1. C. A. Mitchell, J. L. Bahr, S. Arepalli, J. M. Tour, R. Krishnamoorti, "Dispersion of functionalized carbon nanotubes in polystyrene," Macromolecules, vol. 35, pp. 8825-8830, 2002

2. P. Chantrenne, J. L. Barrat, "Analytical model for the thermal conductivity of nanostructures," Suprelattice and Microstructure, vol. 35, pp. 173-186, 2004

3. T. B. Lewis, L. E. Nielsen, "Dynamic mechanical properties of particulate-filled composites," J. Applied Polymer Science, vol. 14, pp. 1449-1471, 1970 
4. Y. S. Song, K. Chung, T. J. Kang, J. R. Youn, "Prediction of permeability tensor for plain woven fabric by using control volume finite element method," Polymers and Polymer Composites, vol. 11, no. 6, pp. 465-476, 2003

5. S. G. Advani, C. L. Tucker III, "The use of tensor to describe and predict fiber orientation in short fiber composites," J. Rheol., vol. 31, no. 8, pp. 751-784, 1987

6. Y. P. Manunya, V. V. Davydenko, P. Pissis, E. V. Lebedev, "Electrical and thermal conductivity of polymers filled with metal powders," European Polym. J., vol. 38, pp. $1887-1897,2002$

7. T. Jeong and A. Ambler, "Power efficiency system for fligth application (PESFA) mission: Low power dissipation in digital circuit design for flgith application/space communications," IEEE Tran. on Aerospace and Electronics System, ISSN: 00189251, 2006 